NORDITA-2000/20 HE

LU TP 00-09

DAPNIA/SPHN-00-11

hep-ph/0003257

January 18, 2019

\title{
Semi-Exclusive Production of Photons at HERA
}

\author{
Paul Hoyer \\ NORDITA \\ Blegdamsvej 17, 2100 Copenhagen, Denmark \\ Martin Maul \\ Department of Theoretical Physics, Lund University \\ Sölvegatan 14 a, S-223 62 Lund, Sweden \\ Andreas Metz \\ DAPNIA/SPhN, CEA-Saclay \\ F-91191 Gif-sur-Yvette, France
}

\begin{abstract}
We study the feasibility of measuring semi-exclusive photon production $\gamma+p \rightarrow \gamma+Y$ at HERA. The cross section of photons produced at large transverse momenta, recoiling off an inclusive system $Y$ of limited mass, can without photon isolation cuts be simply expressed in terms of hard PQCD subprocesses and standard target parton distributions. With the help of event generators we identify the kinematic region where quark and gluon fragmentation processes can be neglected. The cross section in this semi-exclusive region is large enough to be measured with an upgraded HERA luminosity of $\mathcal{L}=100 \mathrm{pb}^{-1}$.

The subprocesses of lowest order in $\alpha_{s}$ are suppressed at low recoil masses $M_{Y}$, compared to higher order gluon exchange, i.e. BFKL contributions. The distinct $M_{Y}$-dependence makes it possible to determine experimentally the kinematic range where the higher order processes dominate.
\end{abstract}

PACS: 12.38.-t, 13.60.-r, 14.70.Bh

Keywords: semi-exclusive reactions, photoproduction, Monte Carlo simulation. 


\section{INTRODUCTION}

Semi-exclusive reactions of the type $A+B \rightarrow C+Y$, where particle $C$ emerges with large transverse momentum and the mass of the inclusive system $Y$ satisfies $\Lambda_{Q C D} \ll M_{Y} \ll E_{c m}$, provide a new tool for probing the structure of hadrons [1]. It is analogous to DIS, $e p \rightarrow e X$, in the sense that particles $A$ and $C$ form an effective current which probes the target $B$ at large momentum transfer $t=\left(p_{A}-p_{C}\right)^{2}$, producing the inclusive system $Y$. Semi-exclusive cross sections factorize into a calculable short distance interaction times a standard target parton $(b)$ distribution $f_{b / B}\left(x_{S},-t\right)$ according to

$$
\frac{d \sigma}{d t d x_{S}}(A+B \rightarrow C+Y)=\sum_{b} f_{b / B}\left(x_{S},-t\right) \frac{d \sigma}{d t}(A b \rightarrow C d),
$$

where

$$
x_{s}=-t /\left(M_{Y}^{2}-t\right)
$$

and the momentum transfer $-t$ serves as factorization scale. For large $-t$ only compact configurations of particles $A$ and $C$ participate in the reaction, and their re-interactions with the target are suppressed. The final parton(s) $d$ merges with the target spectators to form the inclusive system $Y$ (Fig. 1a).
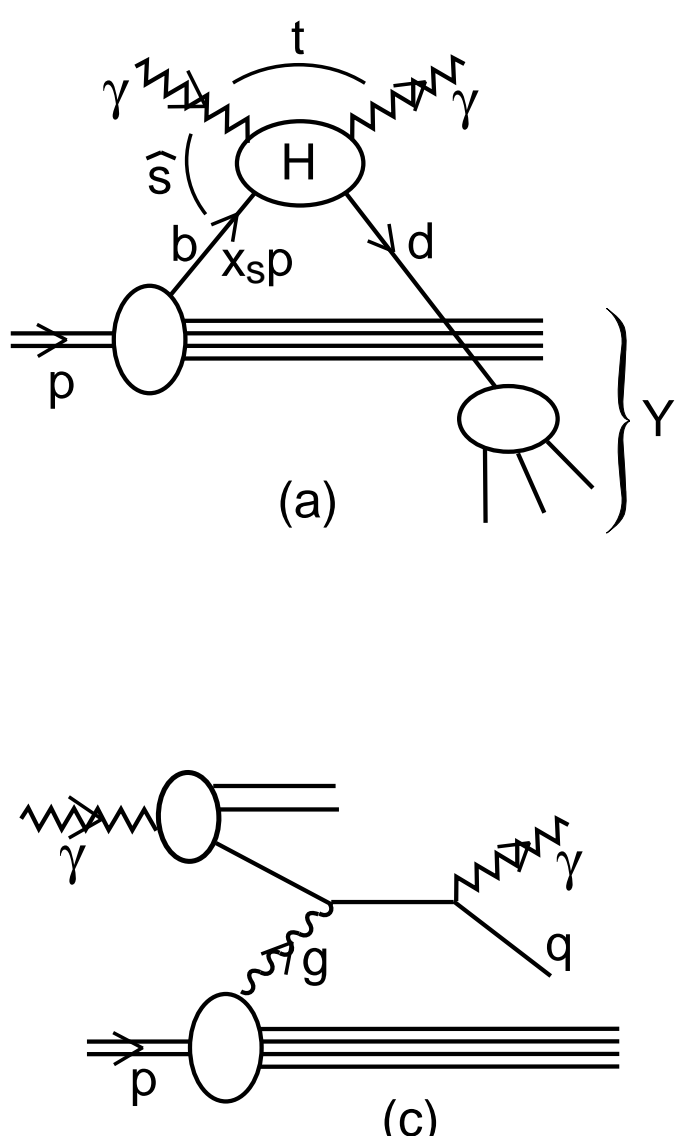

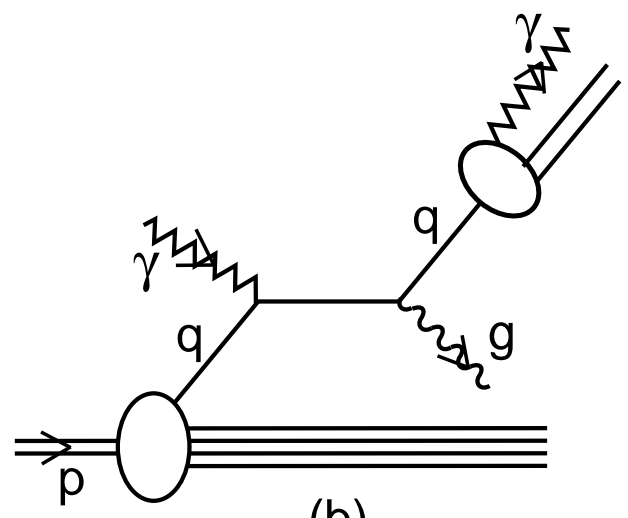

(b)

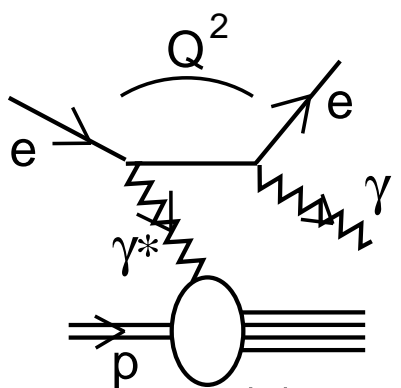

(d)

FIG. 1. (a) Semi-exclusive photoproduction of photons. The hard process H is calculable in PQCD. (b) Photons produced via parton fragmentation are accompanied by comoving hadrons. (c) The resolved photon leaves remnant hadrons in the beam direction. (d) The Bethe-Heitler process is not enhanced at low momentum transfer $Q^{2}$ between the electrons. 
We shall discuss the feasibility of measuring semi-exclusive photon photoproduction, $\gamma p \rightarrow \gamma Y$ at HERA. Here the incoming and outgoing photons together form an effective current which probes a parton (quark or gluon) in the target with resolution $-t$ (Fig. 1a). This picture is valid provided that the final photon emerges isolated from all hadrons in the inclusive system $Y$, which is ensured by the kinematic requirement $W^{2} / M_{Y}^{2} \gg 1$, where $W$ is the total $\gamma p$ center of mass energy, and $M_{Y}$ is the mass of the hadronic system $Y$. Scattering on a single parton implies large $-t$, typically of order $M_{Y}^{2}$. As we shall see, luminosity limitations impose $-t \ll M_{Y}^{2}$ at HERA, implying small $x_{s}$ in Eq. (2).

It is not a priori obvious how stringent the kinematic restrictions need to be to ensure semi-exclusive dynamics. At moderate $W^{2} / M_{Y}^{2}$, photons may be produced via parton fragmentation, following a hard process like $\gamma q \rightarrow g q$ (Fig. 1b). Such a process will typically generate hadrons comoving with the photon, implying $W^{2} / M_{Y}^{2} \sim 1$. Depending on the probability that the photon takes most of the momentum of the fragmenting parton such processes can nevertheless be significant at moderate values of $W^{2} / M_{Y}^{2}$. Based on the PYTHIA [2] and LUCIFER [3] Monte Carlo generators we conclude that fragmentation processes are insignificant for $W^{2} / M_{Y}^{2} \gtrsim 10$ and $-t \gtrsim 4 \mathrm{GeV}^{2}$.

Resolved photon contributions (Fig 1c) are analogously suppressed since hadrons in the beam direction must carry little energy for $W^{2} / M_{Y}^{2}$ to be large. This requires the beam parton to carry nearly all the photon energy, and not to emit collinear bremsstrahlung prior to its hard scattering. The pointlike photon component transfers the full beam energy into the hard process and will thus dominate at large $W^{2} / M_{Y}^{2}$ and $-t$.

We also estimate the contribution of the Bethe-Heitler process (Fig. 1d), where the beam photon is virtual and the final photon is emitted from the electron. This process is typically detected because of a large angle recoil of the electron. We find that the Bethe-Heitler process is in any case negligible for invariant momentum transfers $Q^{2} \lesssim 0.1 \mathrm{GeV}^{2}$ between the initial and final electrons.

HERA data [4.5] on photon production at large transverse momentum have previously been compared with QCD calculations [6] in terms of an isolation cut [9 11], which imposes low accompanying transverse energy in a cone around the photon direction. Such a cut removes a large fraction of the photons produced by parton fragmentation, but allows resolved photon contributions. The isolation procedure requires modelling the non-perturbative fragmentation process. This is avoided for the semi-exclusive cross section (1), which depends only on standard structure functions and perturbatively calculable quantities.

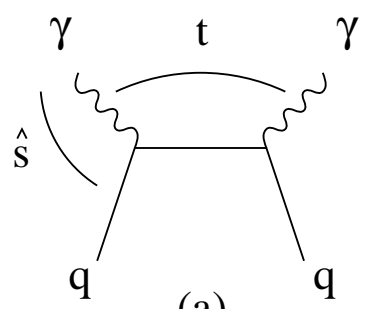

(a)

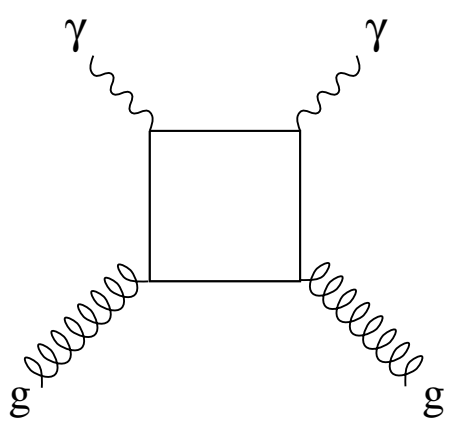

(b)

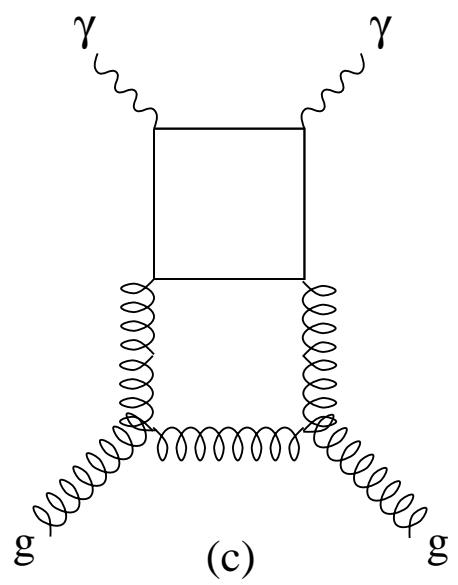

FIG. 2. Compton scattering subprocesses: Low order scattering on a quark (a) and on a gluon (b). At high $\hat{s} /-t$, higher order amplitudes like (c) dominate, due to gluon exchange in the $t$-channel. 
Low order PQCD subprocess amplitudes which contribute to the hard vertex $H$ of Fig. 1a are shown in Fig. 2. It is important to notice that the lowest order contributions of Fig. 2a,b actually are suppressed in the Regge limit $\hat{s}=x_{s} W^{2} \rightarrow \infty$ at fixed (but large) $-t$, which is relevant for semi-exclusive dynamics. A (dimensionless) amplitude involving the exchange of two particles of spins $j_{1}$ and $j_{2}$ in the $t$-channel behaves in the Regge limit as $\sim(\hat{s} /-t)^{\alpha}$, where $\alpha=j_{1}+j_{2}-1$. Hence the contribution of Fig. 2b, with $j_{1}=j_{2}=1 / 2$, has $\alpha=0$. The amplitude of Fig. 2a also has $\alpha=0$. On the other hand, the higher order amplitude of Fig. $2 \mathrm{c}$ has $j_{1}=j_{2}=1$ and thus $\alpha=1$. At even higher orders logarithmic corrections from repeated gluon ladders are expected to build up hard Pomeron exchange in the BFKL approximation, as has been studied extensively for the present process 12 15.

It is interesting to explore at what value of $\hat{s} /-t$ the higher order diagrams begin to dominate. We find that the BFKL amplitude is numerically important compared to the lowest order contributions in the whole semi-exclusive range $\hat{s} /-t \gtrsim 10$. If the BFKL approximation is reliable (or the amplitude of Fig. 2 2 dominates) at such moderate values of the subprocess energy then the subprocess cross section will be found to grow (or be constant) in this whole range. Alternatively, if the lowest order diagrams dominate, the subprocess cross section will decrease in the lower range of $\hat{s} /-t$. This would make it smaller than the BFKL estimate at high $\hat{s} /-t$.

\section{SUBPROCESS CROSS SECTIONS}

The lowest order cross section for $\gamma q \rightarrow \gamma q$ (Fig. 2a) is 16

$$
\frac{d \sigma_{\mathrm{LO}}}{d t}(\gamma q \rightarrow \gamma q)=\frac{2 \pi e_{q}^{4} \alpha^{2}}{\hat{s}^{2}}\left(\frac{\hat{s}}{-\hat{u}}+\frac{-\hat{u}}{\hat{s}}\right)
$$

with $\alpha=e^{2} / 4 \pi$. For the lowest order $\gamma g \rightarrow \gamma g$ process (Fig. 2b b ) wave analogously [17, 18],

$$
\frac{d \sigma_{\mathrm{LO}}}{d t}(\gamma g \rightarrow \gamma g)=2\left(\sum_{q} e_{q}^{2}\right)^{2} \frac{\alpha^{2} \alpha_{s}^{2}}{64 \pi \hat{s}^{2}}\left[\left|M_{1}(\hat{s}, t, \hat{u})\right|^{2}+\left|M_{1}^{\prime}(\hat{u}, t, \hat{s})\right|^{2}+\left|M_{1}^{\prime}(t, \hat{s}, \hat{u})\right|^{2}+20\right],
$$

where

$$
\begin{aligned}
& \left|M_{1}(\hat{s}, t, \hat{u})\right|^{2}=\left(2+2 \frac{t-\hat{u}}{\hat{s}} \ln \frac{t}{\hat{u}}+\frac{t^{2}+\hat{u}^{2}}{\hat{s}^{2}}\left[\ln ^{2} \frac{t}{\hat{u}}+\pi^{2}\right]\right)^{2}, \\
& \left|M_{1}^{\prime}(\hat{u}, t, \hat{s})\right|^{2}=\left(2+2 \frac{\hat{s}-t}{\hat{u}} \ln \frac{\hat{s}}{-t}+\frac{\hat{s}^{2}+t^{2}}{\hat{u}^{2}} \ln ^{2} \frac{\hat{s}}{-t}\right)^{2}+4 \pi^{2}\left(\frac{\hat{s}^{2}+t^{2}}{\hat{u}^{2}} \ln \frac{\hat{s}}{-t}+\frac{\hat{s}-t}{\hat{u}}\right)^{2} .
\end{aligned}
$$

It is interesting to compare the size of the BFKL cross section to the LO ones given above. The $\gamma g \rightarrow \gamma g$ BFKL cross section is to leading logarithmic accuracy given by 13,15

$$
\begin{aligned}
\frac{d \sigma_{\mathrm{BFKL}}}{d t}(\gamma g \rightarrow \gamma g) & =\frac{81}{16} \frac{\alpha^{2} \alpha_{s}^{4}}{576 t^{2}} \pi\left(\sum_{q} e_{q}^{2}\right)^{2}\left[G\left(\frac{3 \alpha_{s}}{\pi} \ln \frac{\hat{s}}{-t}\right)\right]^{2}, \quad \text { with } \\
G(z) & =\int_{-\infty}^{\infty} \frac{d \nu}{1+\nu^{2}} \frac{\nu^{2}}{\left(\nu^{2}+\frac{1}{4}\right)^{2}} \frac{\tanh (\pi \nu)}{\pi \nu} 2\left(11+12 \nu^{2}\right) \exp [z \chi(\nu)] \\
\chi(\nu) & =2 \psi(1)-2 \operatorname{Re}\left[\psi\left(\frac{1}{2}+i \nu\right)\right]
\end{aligned}
$$

In Fig. 3 we show the dimensionless lowest order cross sections $\hat{s}^{2} d \sigma / d t$ for $\gamma u \rightarrow \gamma u$ (solid line) and $\gamma g \rightarrow \gamma g$ (times 10, dot-dashed line). The small size of the lowest order $\gamma g \rightarrow \gamma g$ cross section means that it will be insignificant also in the physical process $\gamma N \rightarrow \gamma Y$, even though the gluon distribution is larger than the quark one at low values of 
$x_{s}$. The dashed line shows the BFKL cross section (6) for $\gamma g \rightarrow \gamma g$, based on iterating higher order diagrams like that in Fig. 2c. We used the fixed value $\alpha_{s}=0.2$ indicated by BFKL fits to HERA and Tevatron data [19 21]. The BFKL cross section is comparable to the lowest order one already at $\hat{s} /-t \simeq 10$, with their ratio growing roughly as $(\hat{s} /-t)^{2}$. It should be stressed, however, that the BFKL analysis is expected to be reliable only for $\hat{s} /-t \gtrsim 100$ [15], and that there may be sizeable corrections from NLO corrections [22].

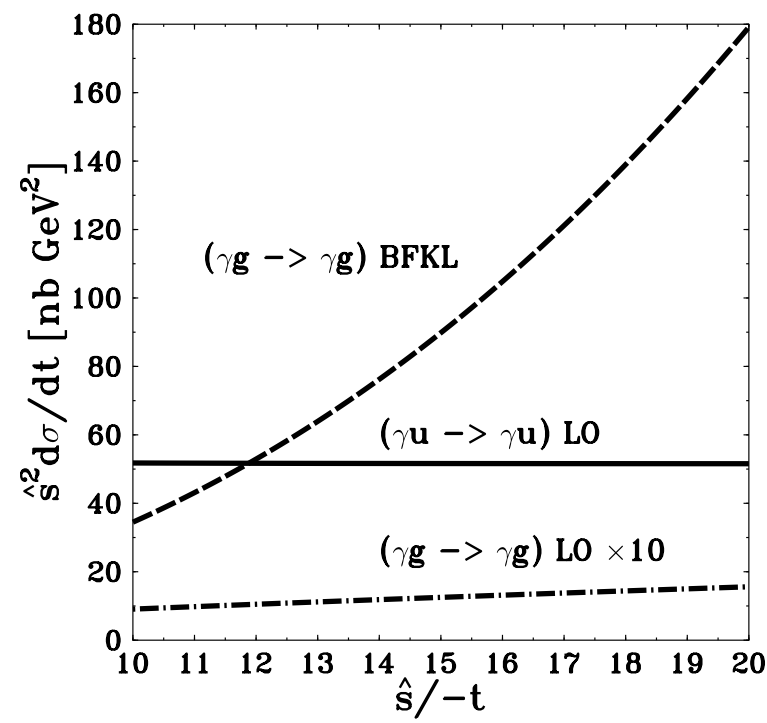

FIG. 3. Lowest order subprocess cross sections $\hat{s}^{2} d \sigma / d t$ for $\gamma u \rightarrow \gamma u$ (solid line) and $\gamma g \rightarrow \gamma g$ (times 10, dot-dashed line) for $10 \leq \hat{s} /-t \leq 20$. The dashed line shows the BFKL cross section extrapolated to this region of moderate subprocess energy, assuming $\alpha_{s}=0.2$. Three active flavors have been used in both the calculation of the process $\gamma g \rightarrow \gamma g$ to LO and the BFKL contribution.

\section{SEMI-EXCLUSIVE PHOTON ELECTROPRODUCTION AT HERA}

The $e p \rightarrow e \gamma Y$ electroproduction cross section is in the semi-exclusive limit $\Lambda_{Q C D}^{2} \ll-t, M_{Y}^{2} \ll W^{2}$ related to the subprocesses through

$$
\frac{d \sigma}{d y d t d x_{s}}(e p \rightarrow e \gamma Y)=f_{\gamma / e}\left(y, Q_{\max }^{2}\right) \sum_{i \in q, g} f_{i / p}\left(x_{s},-t\right) \frac{d \sigma}{d t}(\gamma i \rightarrow \gamma i) .
$$

Here $y$ is the fraction of momentum that the photon, of virtuality $\leq Q_{\max }^{2}$, takes of the electron beam. The WeizsäckerWilliams function $f_{\gamma / e}\left(y, Q_{\max }^{2}\right)$ is given in [23],

$$
f_{\gamma / e}\left(y, Q_{\max }^{2}\right)=\frac{\alpha}{2 \pi}\left[\frac{\left(1+(1-y)^{2}\right)}{y} \ln \frac{Q_{\max }^{2}(1-y)}{m_{e}^{2} y^{2}}+2 m_{e}^{2} y\left(\frac{1}{Q_{\max }^{2}}-\frac{1-y}{m_{e}^{2} y^{2}}\right)\right] .
$$

In our kinematical range the Weizsäcker-Williams scale is identical to the upper limit of the momentum transfer $Q_{\max }^{2}$ between the electrons, as the hard scale $-t$, which characterizes the production process, is much larger than $Q_{\max }^{2}$. A detailed discussion of the choice of the Weizsäcker-Williams scale can be found in Refs. 23,24]. For the parton distributions $f_{i / p}\left(x_{s},-t\right)$ we use the GRV94 LO parameterizations [25].

In this section we first estimate the kinematic region in which the semi-exclusive production mechanism dominates, and then evaluate the physical cross section in this region. 


\section{A. Background from Fragmentation and Decay in Photoproduction}

Large transverse momentum photons can be produced through quark and gluon fragmentation $q, g \rightarrow \gamma+X$, following standard hard scattering processes such as photon gluon fusion $\gamma g \rightarrow q \bar{q}$ and gluon bremsstrahlung $\gamma q \rightarrow q g$. A second source of contributions are photons from hadronic decays like $\pi^{0} \rightarrow \gamma \gamma$. In the following we call both background contributions 'fragmentation'. Such processes typically give hadrons in the photon direction, and are thus suppressed in the limit where $M_{Y}^{2} \ll W^{2}$.

We have used the Monte Carlo event generators PYTHIA [2] and LUCIFER [3] to estimate the range of $W^{2} / M_{Y}^{2}$ in which semi-exclusive production dominates over fragmentation. These event generators include the direct (semiexclusive) $\gamma q \rightarrow \gamma q$ cross section only at lowest order. As we observed in the introduction, the higher order contribution of Fig. 2c (and its possible BFKL enhancement) actually dominates the lowest order process (Fig. 2a) at high $\hat{s} /-t$. Hence comparing the fragmentation background to only the LO semi-exclusive cross-section gives a conservative estimate of the kinematic region where semi-exclusive dynamics dominates. It should also be kept in mind that the fragmentation contribution can be reduced with the help of photon isolation cuts [4, 5, 9-11].

We evaluate the contributions from the direct process $\gamma q \rightarrow \gamma q$ and that from the background $\gamma g \rightarrow q \bar{q}$ and $\gamma q \rightarrow q g$ processes separately. If the final state contains several photons, we choose the one with largest energy $E_{\gamma}^{C M}$ in the CM frame of the photon and the nucleon. The invariant mass $M_{Y}^{2}$ of the remaining particles is then given by $M_{Y}^{2}=W^{2}-2 W E_{\gamma}^{C M}$.
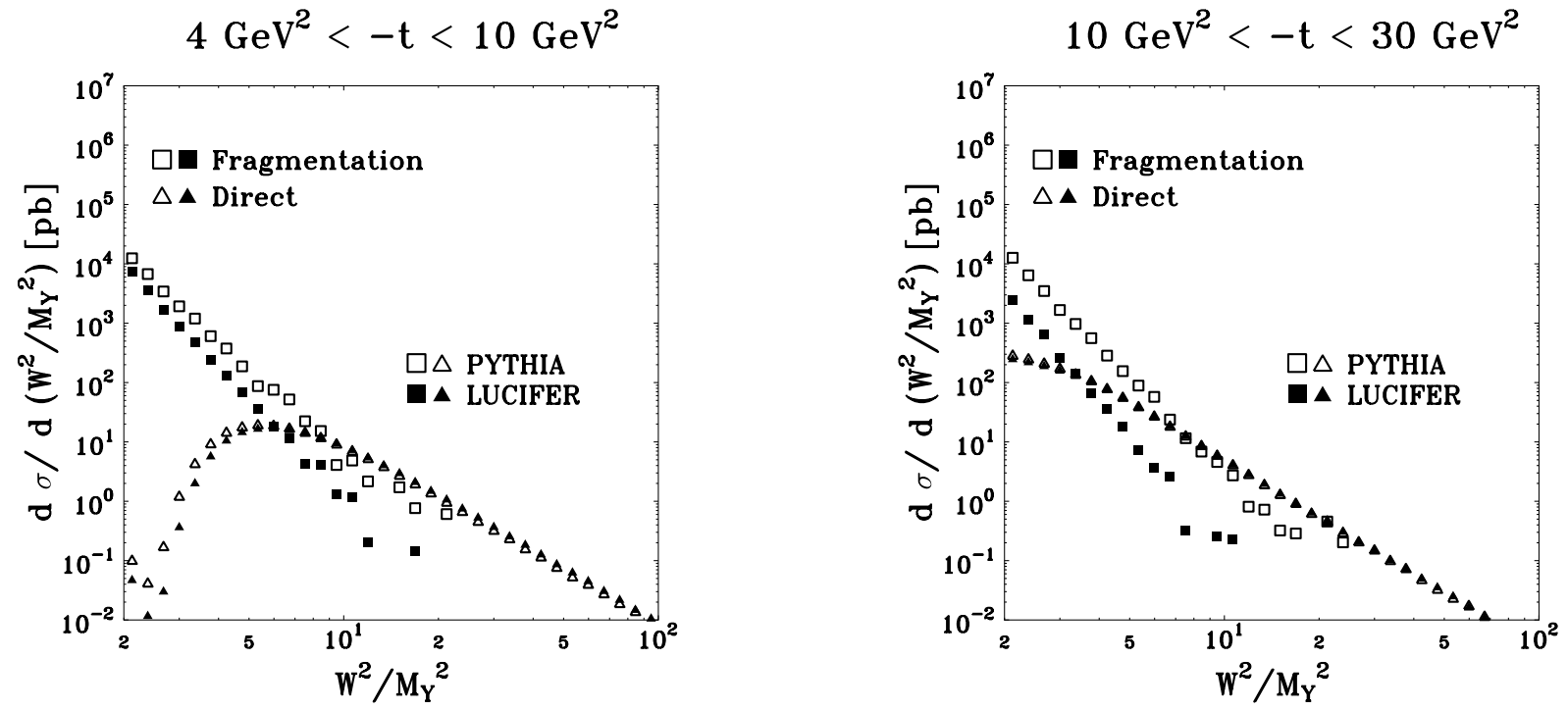

FIG. 4. Inclusive cross sections $d \sigma(\gamma p \rightarrow \gamma Y) / d\left(W^{2} / M_{Y}^{2}\right)$ for the photon with the largest $E_{\gamma}^{C M}$ per event at $W=200 \mathrm{GeV}$. The $t$-range is $4 \mathrm{GeV}^{2}<-t<10 \mathrm{GeV}^{2}$ (left), and $10 \mathrm{GeV}^{2}<-t<30 \mathrm{GeV}^{2}$ (right). The triangles show the direct $(\gamma q \rightarrow \gamma q)$ contribution of Eq. (3) and the squares show photons generated by fragmentation. The open squares and triangles are the result of the PYTHIA program and the filled squares and triangles are from the LUCIFER run. An additional cut .001 $<x_{s}<.7$ has been applied in both figures. Higher order parton showering and resolved photon contributions are not included.

In Fig. 1 we show the PYTHIA and LUCIFER results for $W=200 \mathrm{GeV}$ and two ranges of photon transverse momentum, $4 \mathrm{GeV}^{2}<-t<10 \mathrm{GeV}^{2}$ and $10 \mathrm{GeV}^{2}<-t<30 \mathrm{GeV}^{2}$. It may be seen that the semi-exclusive process $\gamma q \rightarrow \gamma q$ starts to dominate for $W^{2} / M_{Y}^{2} \gtrsim 10$. We have checked that the same conclusion holds for $W=80 \mathrm{GeV}$. Note that for $M_{Y}^{2} \gg-t$, the subprocess variable $\hat{s} /-t$ of Fig. 3 is in the semi-exclusive limit simply related to $W^{2} / M_{Y}^{2}$, 


$$
\frac{W^{2}}{M_{Y}^{2}}=\frac{\hat{s}}{-t}\left(1-\frac{t}{M_{Y}^{2}}\right) \simeq \frac{\hat{s}}{-t}
$$

\section{B. Background from the Bethe-Heitler Process in Electroproduction}

When the incoming photon is virtual the final photon may be radiated off the electron in the Bethe-Heitler $(\mathrm{BH})$ process of Fig. 5 a. We wish to determine the maximum value of $Q^{2}$ for which the $\mathrm{BH}$ cross section can be neglected compared to that of photon emission from the quark, i.e., the Virtual Compton Scattering (VCS) process of Fig. 司b.

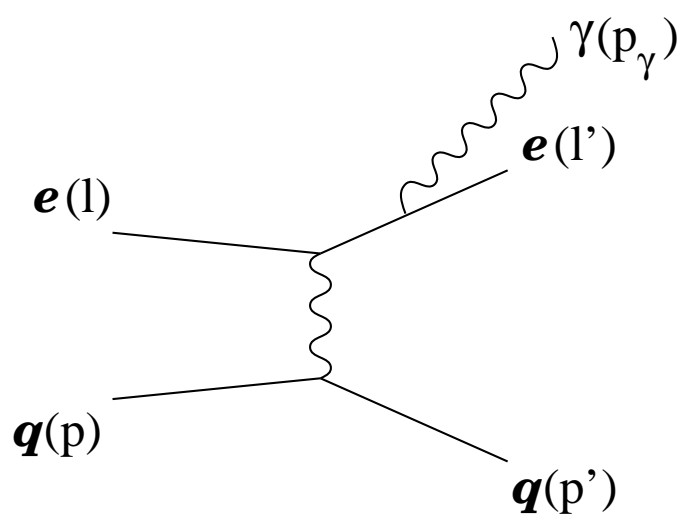

(a)

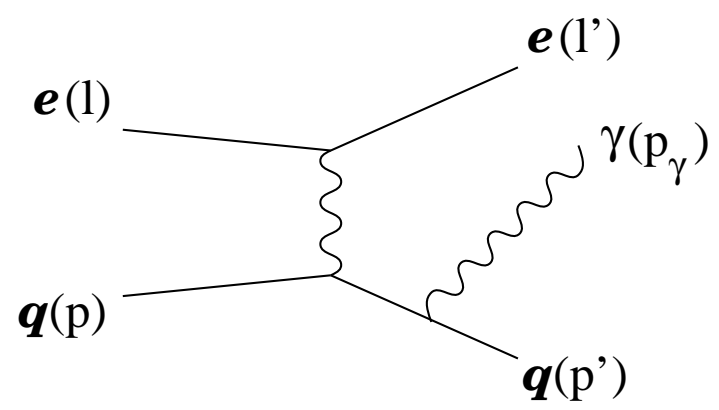

(b)

FIG. 5. Sample diagrams for photon production via the Bethe-Heitler (BH) process (a) and Virtual Compton Scattering (VCS) (b).

At finite $Q^{2}$ the lowest order $e p \rightarrow e \gamma Y$ cross section can be written

$$
\begin{aligned}
\frac{d \sigma_{L O}(e p \rightarrow e \gamma Y)}{d x_{s} d W^{2} d Q^{2} d t d \phi} & =\frac{1}{2(4 \pi)^{4} x_{s} W_{e p}^{4}\left(\hat{s}+Q^{2}\right)} \sum_{q} f_{q / p}\left(x_{s},-t\right) \overline{\left|\mathcal{M}_{q}\right|^{2}}, \quad \text { with } \\
\overline{\left|\mathcal{M}_{q}\right|^{2}} & =\overline{\left|\mathcal{M}_{q}^{V C S}\right|^{2}}+\overline{\left|\mathcal{M}_{q}^{B H}\right|^{2}}+\overline{2 \operatorname{Re}\left(\mathcal{M}_{q}^{B H} \mathcal{M}_{q}^{V C S^{*}}\right)} .
\end{aligned}
$$

Here $Q^{2}=-q^{2}=-\left(l-l^{\prime}\right)^{2}$ and $\hat{s}=(q+p)^{2}=x_{s} W_{\gamma p}^{2}$ in the notation of Fig. 5 . $\phi$ is the angle between $\vec{q} \times \vec{p}_{\gamma}$ and $\vec{\ell} \times \overrightarrow{\ell^{\prime}}$ with the three-vectors given in the $C M$ frame of the virtual photon and the proton, while $W_{e p} \approx 300 \mathrm{GeV}$ is the ep $C M$ energy. We also define

$$
\hat{s}_{l}=\left(l^{\prime}+p_{\gamma}\right)^{2}, \quad \hat{u}_{l}=\left(l-p_{\gamma}\right)^{2}
$$

which can be obtained from $\hat{s}$ and $\hat{u}$ by replacing the quark momentum $p\left(p^{\prime}\right)$ with the lepton momentum $l\left(l^{\prime}\right)$.

The squared VCS and BH matrix elements can now be written as

$$
\begin{aligned}
\overline{\left|\mathcal{M}_{q}^{V C S}\right|^{2}} & =\frac{-4(4 \pi \alpha)^{3} e_{q}^{4}}{Q^{2} \hat{s} \hat{u}} F \\
\overline{\left|\mathcal{M}_{q}^{B H}\right|^{2}} & =\frac{4(4 \pi \alpha)^{3} e_{q}^{2}}{t \hat{s}_{l} \hat{u}_{l}} F
\end{aligned}
$$

with the common factor $F$ given by

$$
F=\hat{s}^{2}+(\hat{s}+t)^{2}+\hat{u}_{l}^{2}+\left(2 W_{e q}^{2}+\hat{u}_{l}\right)^{2}-2 \hat{s}\left(2 W_{e q}^{2}+\hat{u}_{l}\right)-2 t\left(W_{e q}^{2}+\hat{u}_{l}\right)+2 Q^{2}\left(2 \hat{s}+t-3 W_{e q}^{2}-2 \hat{u}_{l}\right)+3 Q^{4},
$$

and $W_{e q}^{2}=(l+p)^{2}=x_{s} W_{e p}^{2}$. 


$$
\begin{aligned}
& \overline{2 \operatorname{Re}\left(\mathcal{M}_{q}^{B H} \mathcal{M}_{q}^{V C S^{*}}\right)}=\frac{8(4 \pi \alpha)^{3} e_{q}^{3}}{Q^{2} t}\left\{Q^{2}\left[-\frac{1}{2}(\hat{s}-\hat{u})\left(\frac{1}{\hat{s}_{l}}-\frac{1}{\hat{u}_{l}}\right)+\frac{1}{\hat{u}_{l}}\left(l \cdot p+l \cdot p^{\prime}\right)+\frac{1}{\hat{s}_{l}}\left(l^{\prime} \cdot p+l^{\prime} \cdot p^{\prime}\right)\right]\right. \\
& +t\left[\frac{1}{2}\left(\hat{s}_{l}-\hat{u}_{l}\right)\left(\frac{1}{\hat{s}}-\frac{1}{\hat{u}}\right)-\frac{1}{\hat{u}}\left(l \cdot p+l^{\prime} \cdot p\right)-\frac{1}{\hat{s}}\left(l \cdot p^{\prime}+l^{\prime} \cdot p^{\prime}\right)\right] \\
& -8\left(l \cdot p l^{\prime} \cdot p^{\prime}+l \cdot p^{\prime} l^{\prime} \cdot p\right)\left(\frac{l \cdot p}{\hat{u} \hat{u}_{l}}+\frac{l \cdot p^{\prime}}{\hat{s} \hat{u}_{l}}+\frac{l^{\prime} \cdot p}{\hat{u} \hat{s}_{l}}+\frac{l^{\prime} \cdot p^{\prime}}{\hat{s} \hat{s}_{l}}\right) \\
& +l \cdot p\left[\frac{\hat{s} \hat{s}_{l}}{\hat{u} \hat{u}_{l}}-3+\frac{2}{\hat{u} \hat{u}_{l}}\left(\hat{s} l^{\prime} \cdot p+\hat{s}_{l} l \cdot p^{\prime}-\left(\hat{u}+\hat{u}_{l}\right) l^{\prime} \cdot p^{\prime}\right)\right] \\
& +l \cdot p^{\prime}\left[\frac{\hat{u} \hat{s}_{l}}{\hat{s} \hat{u}_{l}}-3+\frac{2}{\hat{s} \hat{u}_{l}}\left(-\hat{s}_{l} l \cdot p-\hat{u} l^{\prime} \cdot p^{\prime}+\left(\hat{s}+\hat{u}_{l}\right) l^{\prime} \cdot p\right)\right] \\
& +l^{\prime} \cdot p\left[\frac{\hat{s} \hat{u}_{l}}{\hat{u} \hat{s}_{l}}-3+\frac{2}{\hat{u} \hat{s}_{l}}\left(-\hat{s} l \cdot p-\hat{u}_{l} l^{\prime} \cdot p^{\prime}+\left(\hat{u}+\hat{s}_{l}\right) l \cdot p^{\prime}\right)\right] \\
& \left.+l^{\prime} \cdot p^{\prime}\left[\frac{\hat{u} \hat{u}_{l}}{\hat{s} \hat{s}_{l}}-3+\frac{2}{\hat{s} \hat{s}_{l}}\left(\hat{u} l \cdot p^{\prime}+\hat{u}_{l} l^{\prime} \cdot p-\left(\hat{s}+\hat{s}_{l}\right) l \cdot p\right)\right]\right\} \text {. }
\end{aligned}
$$

The results presented in Eqs. (12) - (15) have previously been computed, e.g., in Ref. 26], but with our particular choice of kinematical variables we obtained more compact expressions.

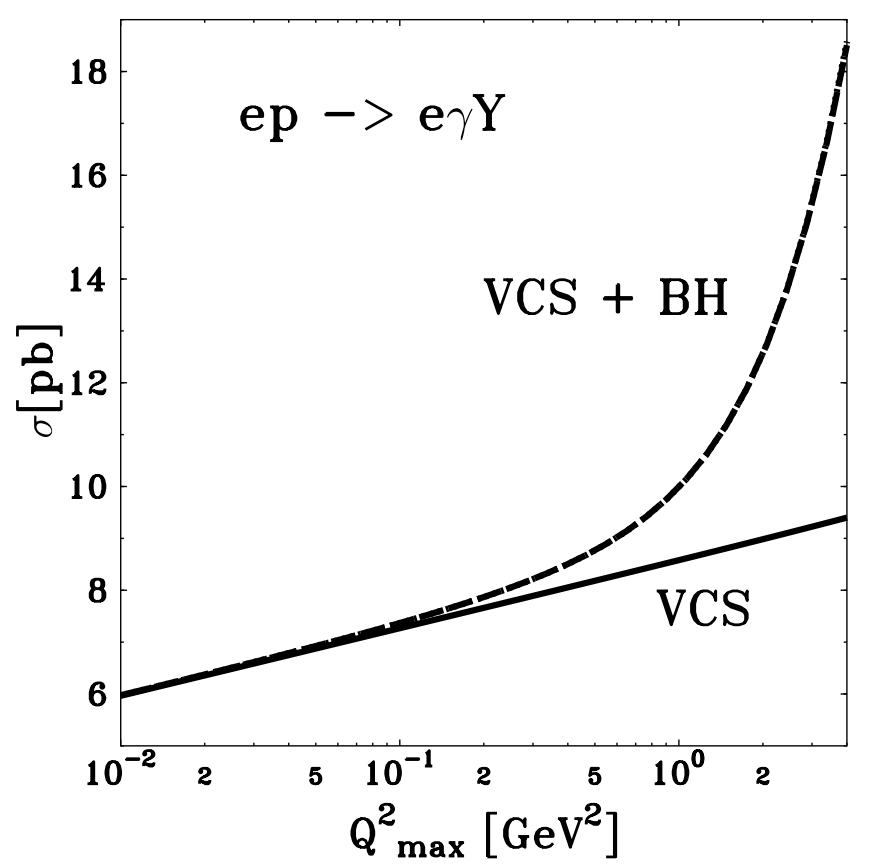

FIG. 6. Dependence of the total $e p \rightarrow e \gamma Y$ cross section on the maximal momentum transfer $Q_{\max }^{2}$. The solid line shows the contribution of Virtual Compton Scattering (VCS, Fig. 5b) alone, while the dashed line includes also the Bethe-Heitler (BH, Fig. 5a) contribution and the interference term. The kinematic region is defined in the text.

In Fig. 6 we compare the VCS to the total VCS+BH cross section for $e p \rightarrow e \gamma Y$ as a function of $Q_{\max }^{2}$, the maximum momentum transfer between the electrons. The differential cross section (10) was integrated over the 
ranges $x_{s} \in[0.01,0.7], W \in[40 \mathrm{GeV}, 160 \mathrm{GeV}], \phi \in[0,2 \pi],-t \in\left[4 \mathrm{GeV}^{2}, 10 \mathrm{GeV}^{2}\right]$, and $Q^{2} \in\left[m_{e}^{2}, Q_{\max }^{2}\right.$ 周

\section{The Semi-Exclusive Cross Section}

We have found that the process $e p \rightarrow e \gamma Y$ can be used to study semi-exclusive photon production at HERA in the kinematic range $W^{2} / M_{Y}^{2} \gtrsim 10, Q^{2} \lesssim 0.1 \mathrm{GeV}^{2}$ and $-t \gtrsim 4 \mathrm{GeV}^{2}$. In Fig. 目 we show the cross section

$$
\frac{d \sigma(e p \rightarrow e \gamma Y)}{d\left(W^{2} / M_{Y}^{2}\right)}=\int_{0.25}^{0.75} d y f_{\gamma / e}\left(y, Q_{\max }^{2}=0.1 \mathrm{GeV}^{2}\right) \int_{|t|_{\min }}^{|t|_{\max }} d t \frac{-t\left(1-x_{s}\right)^{2}}{W^{2}} \sum_{i \in q, g} f_{i / p}\left(x_{s},-t\right) \frac{d \sigma}{d t}(\gamma i \rightarrow \gamma i)
$$

for two ranges of momentum transfer, $4 \mathrm{GeV}^{2}<-t<10 \mathrm{GeV}^{2}$ and $10 \mathrm{GeV}^{2}<-t<30 \mathrm{GeV}^{2}$. The full curve shows the contribution of the LO process of Eq. (3) (Fig. 2a). The dashed curve shows the contribution of the BFKL subprocess of Eq. (6) (Fig. 2 2c, plus gluon ladder iterations), together with the corresponding $\gamma q \rightarrow \gamma q$ sea quark BFKL contribution [14],

$$
\frac{d \sigma_{\mathrm{BFKL}}}{d x_{s} d t}=\left[g\left(x_{s},-t\right)+\frac{16}{81} \Sigma\left(x_{s},-t\right)\right] \frac{d \sigma_{\mathrm{BFKL}}}{d t}(\gamma g \rightarrow \gamma g)
$$
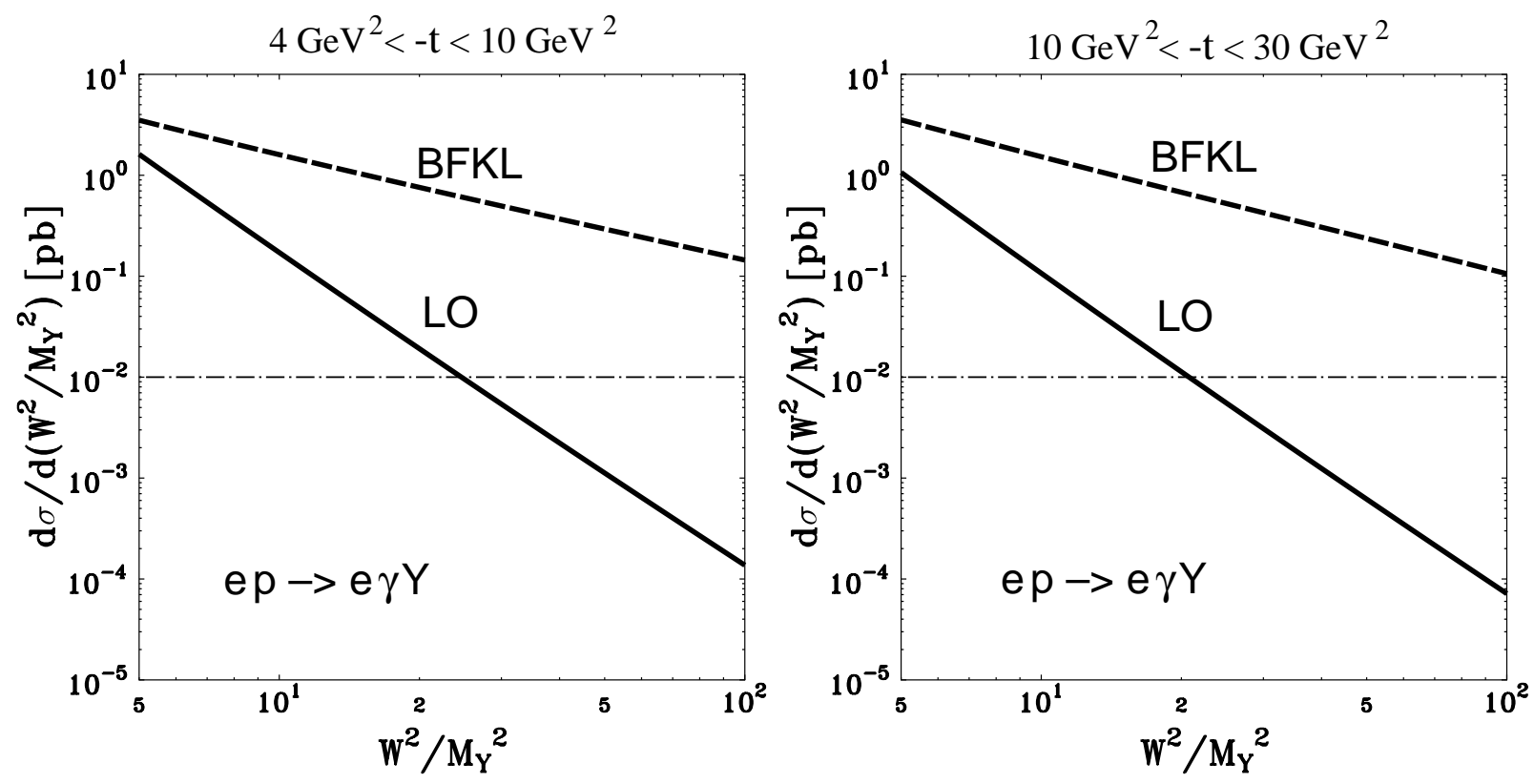

FIG. 7. The cross section $d \sigma(e p \rightarrow e \gamma Y) / d\left(W^{2} / M_{Y}^{2}\right)$ of Eq. (16) shown for two ranges of $t: 4 \mathrm{GeV}^{2}<-t<10 \mathrm{GeV}^{2}$ (left) and $10 \mathrm{GeV}^{2}<-t<30 \mathrm{GeV}^{2}$ (right). The solid line shows the contribution of the LO process of Eq. (3) (Fig. 2a). The dashed line shows that of the BFKL process of Eq. (17) (Fig. 2c plus gluon ladder iterations). The horizontal dash-dotted line gives the 1 event level per unit of $W^{2} / M_{Y}^{2}$, for a HERA luminosity of 100 events/pb.

\footnotetext{
${ }^{*}$ In order to ensure that the electron propagators are far off-shell we also required $\hat{s}_{l},-\hat{u}_{l}>-t_{\min }=4 \mathrm{GeV}^{2}$. This cut is, however, irrelevant for $Q^{2} \lesssim 1 \mathrm{GeV}^{2}$.
} 
where $\Sigma$ denotes the quark singlet distribution $\Sigma=\sum_{q}\left[f_{q / p}+\bar{f}_{q / p}\right]$. We use $\alpha_{s}=0.2$ in the BFKL cross section and assume three (four) active flavors in the low (high) $|t|$-range. The horizontal dash-dotted line indicates the 1 event level per unit of $W^{2} / M_{Y}^{2}$, given a nominal HERA luminosity of 100 events/pb.

We should emphasize that the BFKL approximation may not be reliable for $W^{2} / M_{Y}^{2} \lesssim 100$ [15], hence the dashed curve is an extrapolation. It is nevertheless interesting to observe that this extrapolation dominates the LO $q \gamma \rightarrow q \gamma$ contribution over the whole semi-exclusive range $W^{2} / M_{Y}^{2} \gtrsim 10$. This conclusion is insensitive to the $t$-range and also to the range of $y$ (not shown).

\section{CONCLUSIONS}

Semi-exclusive processes $A+B \rightarrow C+Y$ provide a new tool for investigating hadron structure. Effective currents formed by the $A \bar{C}$ system generalize the virtual photon probe familiar from DIS and can carry charge, flavor, baryon and other quantum numbers [1]. Before this tool can be put to use, at least two questions need to be answered:

(i) How stringent limits $\Lambda_{Q C D}^{2} \ll-t, M_{Y}^{2} \ll W^{2}$ must be imposed in order for the semi-exclusive production mechanism to dominate?

(ii) Can the hard $A \bar{C}$ vertex be reliably computed using $P Q C D$ ?

In this paper we studied the process $\gamma p \rightarrow \gamma Y$, which is especially simple in the sense that both particles $A$ and $C$ have a point like component. Based on simulations with the PYTHIA and LUCIFER event generators we concluded that semi-exclusive dynamics should dominate for $W^{2} / M_{Y}^{2} \gtrsim 10$ and $-t \gtrsim 4 \mathrm{GeV}^{2}$. Photon emission from the electron (the Bethe-Heitler process) is insignificant for incoming photon virtualities $Q^{2} \lesssim 0.1 \mathrm{GeV}^{2}$, and can be further suppressed with angular cuts. The semi-exclusive cross section should be measurable at HERA, assuming that the subprocess cross section $\hat{\sigma}(\gamma q \rightarrow \gamma q)$ is not smaller than its lowest order (LO) PQCD approximation.

Point (ii) above is non-trivial, since the semi-exclusive kinematics implies a high energy (Regge) limit for the subprocess, $\hat{s} /-t \gg 1$. Little is known about the importance of higher order (HO) PQCD corrections in this limit. In the process under study the situation is particularly intriguing since the LO subprocess diagrams shown in Fig. 2aa,b correspond to $q \bar{q}$ exchange in the $t$-channel. At high subenergies $\hat{s}$ they are therefore suppressed by a factor $1 / \hat{s}^{2}$ in the cross section compared to the $\mathcal{O}\left(\alpha_{s}^{4}\right)$ gluon exchange contribution of Fig. 2k. The latter is, on the other hand, just the first term in the series of gluon ladder diagrams which is supposed to build up the BFKL Pomeron in this process 13 15.

It is not clear from which value of $\hat{s} /-t \mathrm{HO}$ contributions like Fig. 2 $2 \mathrm{c}$ start to dominate the LO processes of Fig. 2a,b. The BFKL approximation has been assumed to be relevant for $\hat{s} /-t \gtrsim 100$ [15]. Extrapolating the BFKL cross section to lower energies we found (Fig. 7) that it would in fact dominate the LO cross section in the whole range of semi-exclusive dynamics, $\hat{s} /-t \gtrsim 10$. The ratio $\sigma_{H O} / \sigma_{L O}$ behaves approximately like $\left(W^{2} / M_{Y}^{2}\right)^{2}$, closely reflecting the $\hat{s} /-t$ dependence of the respective subprocesses, $c f$. Eq. (9). A measurement of the $\left(W^{2} / M_{Y}^{2}\right)^{2}$ dependence of the cross section will thus directly determine the nature of the dominant $t$-channel exchange.

We conclude that the large HERA energy in principle allows accessing the semi-exclusive kinematic region, with its double hierarchy of large scales. The limiting factor will be the luminosity. If the cross section is approximately given by the lowest order contribution in Fig. 7 then only a restricted range of $\left(W^{2} / M_{Y}^{2}\right)^{2}$ can be studied. Since the higher order two gluon exchange (BFKL) contributions fall off more slowly with $\left(W^{2} / M_{Y}^{2}\right)^{2}$ they will eventually dominate. If their normalization is even close to that indicated by the BFKL extrapolation of Fig. 7 there should be a rich semi-exclusive phenomenology at HERA, not only for photon but also for meson $(\pi, \rho, J / \psi)$ production. Further work is needed to estimate the feasibility of measuring charge exchange processes and the three gluon (Odderon) contribution to $\pi^{0}$ production.

Acknowledgements. We are grateful for helpful discussions with Stan Brodsky and Markus Diehl. This work is supported in part by the EU/TMR contract EBR FMRX-CT96-0008. 
[1] S.J. Brodsky, M. Diehl, P. Hoyer and S. Peigne, Phys. Lett. B449 (1999) 306, hep-ph/9812277.

For earlier related work, see C.E. Carlson and A.B. Wakely, Phys. Rev. D48 (1993) 2000.

[2] T. Sjöstrand, Comput. Phys. Commun. 82 (1994) 74.

[3] G. Ingelman and A. Weigend, Comput. Phys. Commun. 46 (1987) 241.

[4] J. Breitweg et al. [ZEUS Collaboration], Phys. Lett. B413 (1997) 201 hep-ex/9708038], and Phys. Lett. B472 (2000) 175 hep-ex/9910045.

[5] S. W. Lee [ZEUS collaboration], Nucl. Phys. Proc. Suppl. 82 (2000) 162 hep-ex/9911051.

[6] D. W. Duke and J.F. Owens, Phys. Rev. D26 (1982) 1600; Erratum: Phys. Rev. D28 (1983) 1227.

[7] P. Aurenche, A. Douiri, R. Baier, M. Fontannaz and D. Schiff, Z. Phys. C24 (1984) 309.

[8] P. Aurenche, P. Chiappetta, M. Fontannaz, J.P. Guillet and E. Pilon, Z. Phys. C56 (1992) 589.

[9] L.E. Gordon, Phys. Rev. D57 (1998) 235, hep-ph/9707464.

[10] M. Krawczyk and A. Zembrzuski, IFT 98/12, hep-ph/9810253.

[11] S. Frixione, Phys. Lett. B429 (1998) 369, hep-ph/9801442.

[12] I. F. Ginzburg and D. Y. Ivanov, Phys. Rev. D54 (1996) 5523 hep-ph/9604437.

[13] D.Y. Ivanov and M. Wüsthoff, Eur. Phys. J. C8 (1999) 107, hep-ph/9808455.

[14] N.G. Evanson and J.R. Forshaw, Phys. Rev. D60 (1999) 034016, hep-ph/9902481.

[15] B.E. Cox and J.R. Forshaw, hep-ph/9912486.

[16] A. Afanasev, C.E. Carlson and C. Wahlquist, Phys. Rev. D58 (1998) 054007, hep-ph/9706522.

[17] M.A. Doncheski and R.W. Robinett, Phys. Rev. D46 (1992) 2011.

[18] E.L. Berger, E. Braaten and R.D. Field, Nucl. Phys. B239 (1984) 52.

[19] J. Bartels, J. R. Forshaw, H. Lotter and M. Wüsthoff, Phys. Lett. B375 (1996) 301 [hep-ph/9601201.

[20] ZEUS Collaboration, Study of the Diffractive Production of Vector Mesons at Large $Q^{2}$ or at high $|t|$ at HERA, Contribution to the International Europhysics Conference on High Energy Physics, Tampere, Finland 1999. See also http://zedy00.desy.de/ schlenst/eps99_paper.html.

[21] B. Cox, J. Forshaw and L. Lönnblad, JHEP 9910 (1999) 023 thep-ph/9908464].

[22] V. S. Fadin and L. N. Lipatov, Phys. Lett. B429 (1998) 127 hep-ph/9802290.

[23] S. Frixione, M.L. Mangano, P. Nason and G. Ridolfi, Phys. Lett. B319 (1993) 339, hep-ph/9310350.

[24] S. Frixione and G. Ridolfi, Nucl. Phys. B507 (1997) 315 hep-ph/9707345].

[25] M. Glück, E. Reya and A. Vogt, Z. Phys. C67 (1995) 433.

[26] S. J. Brodsky, J. F. Gunion and R. Jaffe, Phys. Rev. D6 (1972) 2487. 